\title{
Gene Screening of Astrocytoma Grade III Relative to Grade II via Network Analysis: A New Molecular Insight
}

\author{
Mostafa Rezaei-Tavirani ${ }^{1,{ }^{*}}$ and Sina Rezaei Tavirani ${ }^{1}$ \\ ${ }^{1}$ Proteomics Research Center, Shahid Beheshti University of Medical Sciences, Tehran, Iran \\ "Corresponding author: Proteomics Research Center, Shahid Beheshti University of Medical Sciences, Tehran, Iran. Email: tavirany@yahoo.com \\ Received 2018 August 12; Revised 2018 October 17; Accepted 2018 October 21.
}

\begin{abstract}
Background: Astrocytomas (sub-group of gliomas) are central neuron system malignant diseases, which are originated from astrocytes. There are several documents about molecular mechanism of astrocytomas and many related genes are introduced. Here, gene expression profile alteration for grade III relative to grade II of astrocytoma is analyzed via protein-protein interaction (PPI) network to screen and introduce critical differentially expressed genes (DEGs).

Methods: The significant DEGs were extracted from gene expression omnibus (GEO) database and included in a PPI network by Cytoscape software. The common significant DEGs and central nodes of the network were selected and enriched by ClueGO to find related biological terms.

Results: Twenty critical genes including zinc finger protein 765 (ZNF765), zinc finger protein 540 (ZNF540), Zeste white 10 (ZW10) ZW10 interacting kinetochore protein (ZWINT), collagen type XVIII alpha 1 chain (COL18A1), protamine 2 (RRM2), kinesin family member 23 (KIF23), minichromosome maintenance 10 (MCM10), anaphase promoting complex subunit 7 (ANAPC7), NADPH oxidase activator 1(NOXA1), ryanodine receptor 2 (RYR2), myozenin 3 (MYOZ3), myosin binding protein C (MYBPC2), fast type, keratin 17 (KRT17), zinc phosphodiesterase ELAC protein 2 (ELAC2), Abelson helper integration site 1 (AHI1), latent transforming growth factor beta binding protein 1 (LTBP1), kaptin (actin binding protein) (KPTN), BEN domain containing 3 (BEND3), cysteine and histidine rich 1 (CYHR1), and hyaluronoglucosaminidase 2 (HYAL2) were identified. Eight class of biological terms related to the critical genes were determined and discussed.

Conclusions: A wide range of the introduced significant genes indicates that there are several possible therapeutic ways to challenge astrocytoma.
\end{abstract}

Keywords: Astrocytoma, Gene, Protein, Grade, Network

\section{Background}

As central neuron system (CNS) cancers, astrocytomas are considered as common glial tumors (1). Grade II (diffused astrocytoma) of this disease is characterized by slow developing tumor, considerable cell differentiation, and affinity to malignancy evolution (2). An investigation indicates that grade II astrocytoma frequently is diagnosed in 35-year-old patients (range of 20 - 45) (3). Anaplastic astrocytoma (grade III) is more malignant glioma than grade II (4). One year and 5 years survival for anaplastic astrocytoma patients is estimated at $75 \%$ and $25 \%$, respectively (5). Low-grade gliomas (grade II gliomas) in adults include 3 groups as astrocytomas, oligodendrogliomas, and oligo-astrocytomas. Differences between the introduced groups are arisen from cellular origination of them. The low-grade disease tends to convert into higher grades (grades III and IV) (6). As it is reported, mutation fre- quency in astrocytoma grade III for isocitrate dehydrogenase 1 (IDH1), alpha-thalassemia/mental retardation syndrome, nondeletion type, X-linked (ATRX), and Tumor protein 53 (TP53) genes are as $75 \%, 63 \%$, and $82 \%$, respectively (7). Details of up-regulation of ribosomal proteins $\mathrm{S} 2,8, \mathrm{~S} 18$, and L37A and down-regulation of apolipoprotein D (APOD), Sortilin-related receptor, L(DLR class) A (SORL1), SPARC (Osteonectin), Cwcv and Kazal like domains proteoglycan 2 (SPOCK2), protease, serine, 11 (PRSS11), DNA-binding protein inhibitor ID-3 (ID3) genes in the higher grades of asrtrocytoma are reported by MacDonald et al. (8). Kunkle et al. investigation displayed that deregulation of collagen type IV A1(COL4A1), epidermal growth factor receptor (EGFR), basic transcription factor 3(BTF3), MAGUK p55 subfamily member 2 (MPP2), RAS oncogene GTPases 31 (RAB31), cyclin-dependent kinase 4 (CDK4), cluster of differentiation 99 (CD99), annexin A2 (ANXA2), DNA topoisomerase 
II alpha (TOP2A), and SERPINE1 mRNA binding protein 1 (SERBP1) genes is related to the high grade of astrocytoma. They showed that except MPP2, the other genes were upregulated (9). Recently, the screening of numerous genes to find restricted numbers of the critical genes, which are involved in diseases via PPI network analysis, has attracted the attention of researchers (10-12). Since the progression of lower grades of astrocytoma to higher grades is accompanied by difficulty of treatment and reduction of survival rate, here DEGs between grades II and III astrocytoma are investigated via PPI network analysis. The critical genes and related biological terms will determine to introduce therapeutic and diagnostic possible biomarkers.

\section{Methods}

Array gene expression profiles were used from series GSE54004 of GPL18281 platform (www.ncbi.nlm.nih.gov/geo/query/acc.cgi?acc=GSE54004). Six grade II astrocytoma patients including 3 males and 3 females and 21 grade III astrocytoma patients including 8 males and 13 females ranged 20 to 50 years old were included in the research.

This platform is recorded by "gene expression data from malignant gliomas" title in GEO database. As it is reported, the samples were formalin-fixed and paraffinembedded. The profiles were matched by box plot examination via GEO2R analysis and the top 250 significant DEGs were determined. The unknown DEGs were excluded and $\mathrm{P}$ value less than 0.05 and fold change (FC) above 2 were considered. The screened DEGs were included to construct PPI network via Cytoscape version 3.6.0 (13). The network was constructed via public database query of Cytoscape and analyzed by "network analyzer", which is an application of Cytoscape. The top nodes based on degree value above mean \pm standard deviation (SD) were identified as hub-genes (14). The common hubs and significant DEGs were marked as critical genes, which distinct grades II and III of astrocytoma. The critical genes were enriched via gene ontology (GO) analysis by CluGO plugin of Cytoscape software (15). The related significant $(\mathrm{P}<0.05)$ biological processes, molecular function, cellular component, and biochemical pathways were determined from all databases, which were connected to ClueGO.

\section{Results}

Comparing grades II and III astrocytoma patient samples, box plot is shown in Figure 1. Based on the midlines, the samples are matched statistically and can be compared. Forty-five significant DEGs were included in the
PPI network. The created network was comprised of 4489 nodes ( 45 query genes plus 4444 related neighbors). Fitted power law of degree distribution (Figure 2) was characterized by $\mathrm{y}=\mathrm{ax}{ }^{\mathrm{b}}$ equation that $\mathrm{a}, \mathrm{b}, \mathrm{R}$-squared, and correlation were $85.147,-0.985,0.637$, and 0.898 , respectively. The R-squared is calculated based on logarithmized values. This mode of correlation consists to the scale-free networks (16). There are limited numbers of nodes in scale-free networks, which are central nodes. PPI networks are usually scale-free network; therefore, few nodes among numerous nodes are distinguishable. The numbers of 50 nodes were determined as hubs of the network. There were 20 common hub-nodes and significant DEGs that were identified as critical genes. Degree and logarithm of FC of the critical genes are illustrated in Table 1 . The significant $(\mathrm{P}<0.05)$ GO enriched properties of the 20 critical genes, which are classified in the 8 groups, are shown in Figure 3.

\section{Discussion}

Since the conversion of low grades to higher grades is accompanied by a reduction of patient's survival rate,

\begin{tabular}{|c|c|c|c|}
\hline $\mathbf{R}$ & Gene Name & Degree & $\log$ FC \\
\hline $\mathbf{1}$ & ZNF765 & 415 & 1.285 \\
\hline 2 & ZNF540 & 401 & -1.168 \\
\hline 3 & ZWINT & 197 & 1.073 \\
\hline 4 & COL18A1 & 132 & -1.137 \\
\hline 5 & RRM2 & 128 & 1.106 \\
\hline 6 & KIF23 & 94 & 1.059 \\
\hline 7 & MCM10 & 87 & 1.161 \\
\hline 8 & ANAPC7 & 76 & 1.021 \\
\hline 9 & NOXA1 & 68 & -1.354 \\
\hline 10 & RYR2 & 68 & -1.026 \\
\hline 11 & MYOZ3 & 67 & -1.113 \\
\hline 12 & MYBPC2 & 63 & -1.345 \\
\hline 13 & KRT17 & 59 & -1.076 \\
\hline 14 & ELAC2 & 51 & 1.074 \\
\hline 15 & AHI1 & 45 & -1.246 \\
\hline 16 & LTBP1 & 45 & 1.038 \\
\hline 17 & KPTN & 39 & -1.034 \\
\hline 18 & BEND3 & 36 & 1.141 \\
\hline 19 & CYHR1 & 32 & 1.026 \\
\hline 20 & HYAL2 & 28 & 1.054 \\
\hline
\end{tabular}

${ }^{\mathrm{a}}$ The genes are sorted by degree value. 


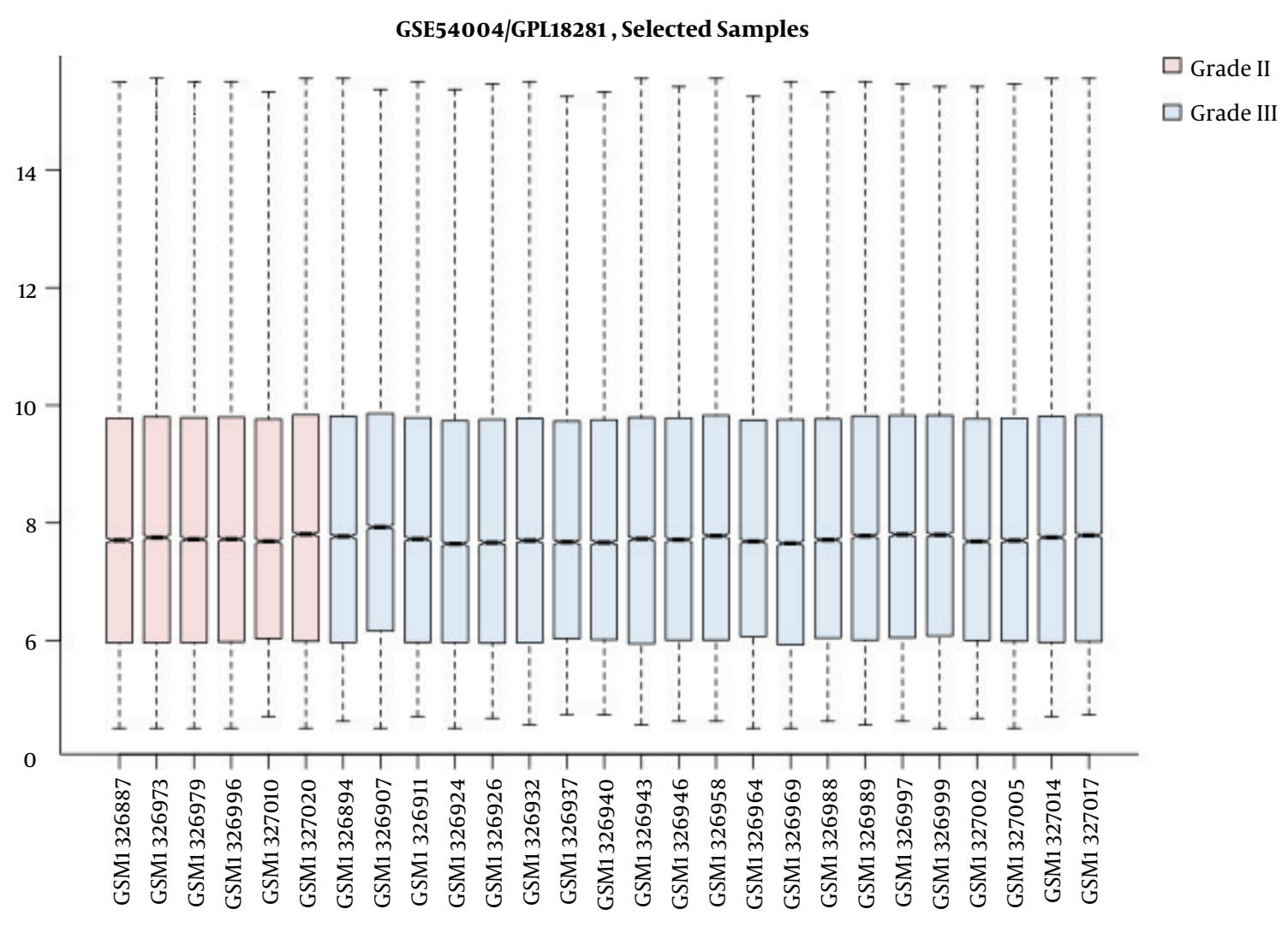

Figure 1. Box plot presentation of gene expression profiles of 6 ( 3 males and 3 females) grade II and 21 ( 8 males and 13 females) grade III astrocytoma patients is illustrated. The patients were aged 20 to 50 years old. Formalin-fixed and paraffin-embedded samples were used.

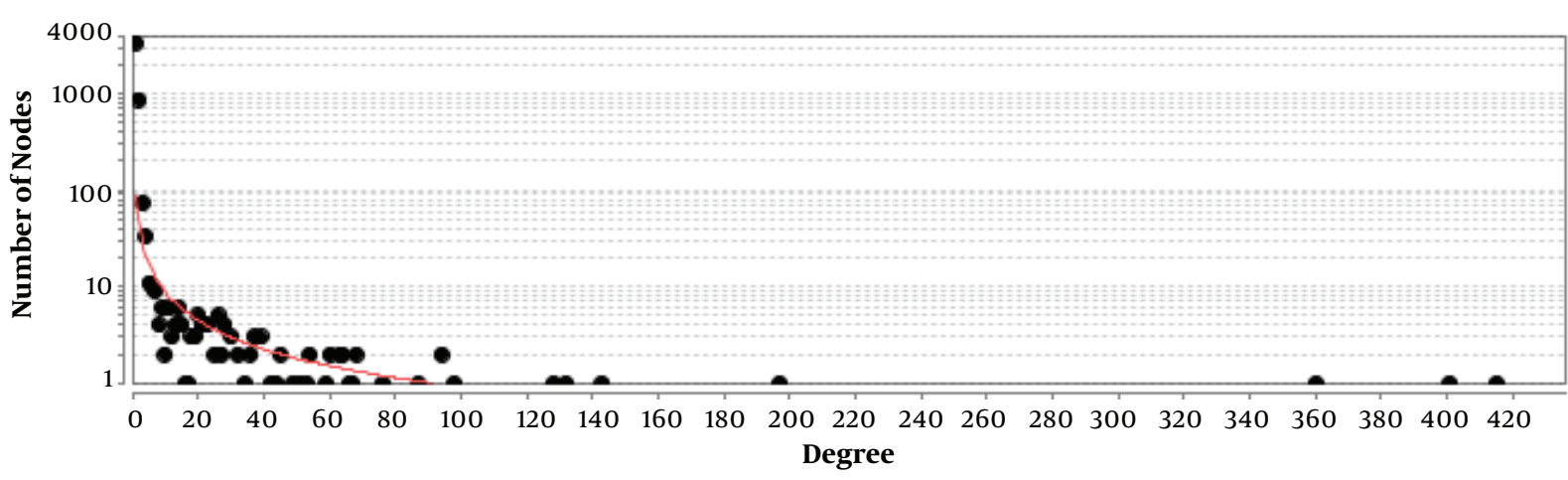

Figure 2. Degree distribution curve of PPI network of grade III astrocytoma versus grade II is represented. The fitted power law is red colored.

a better understanding of molecular features of this transition is an important process. In this regard, PPI network analysis is a suitable method to highlight the critical and effective genes related to conversion of grade II to III of astrocytoma. As depicted in the Figure 1, the pa- tient's gene expression profiles of the two groups (grades II and III) are comparable. Among numerous DEGs, only 45 genes were significantly investigable. PPI network analysis showed without considering the neighbors and relevant genes, the 45 DEGs cannot construct a network (the 


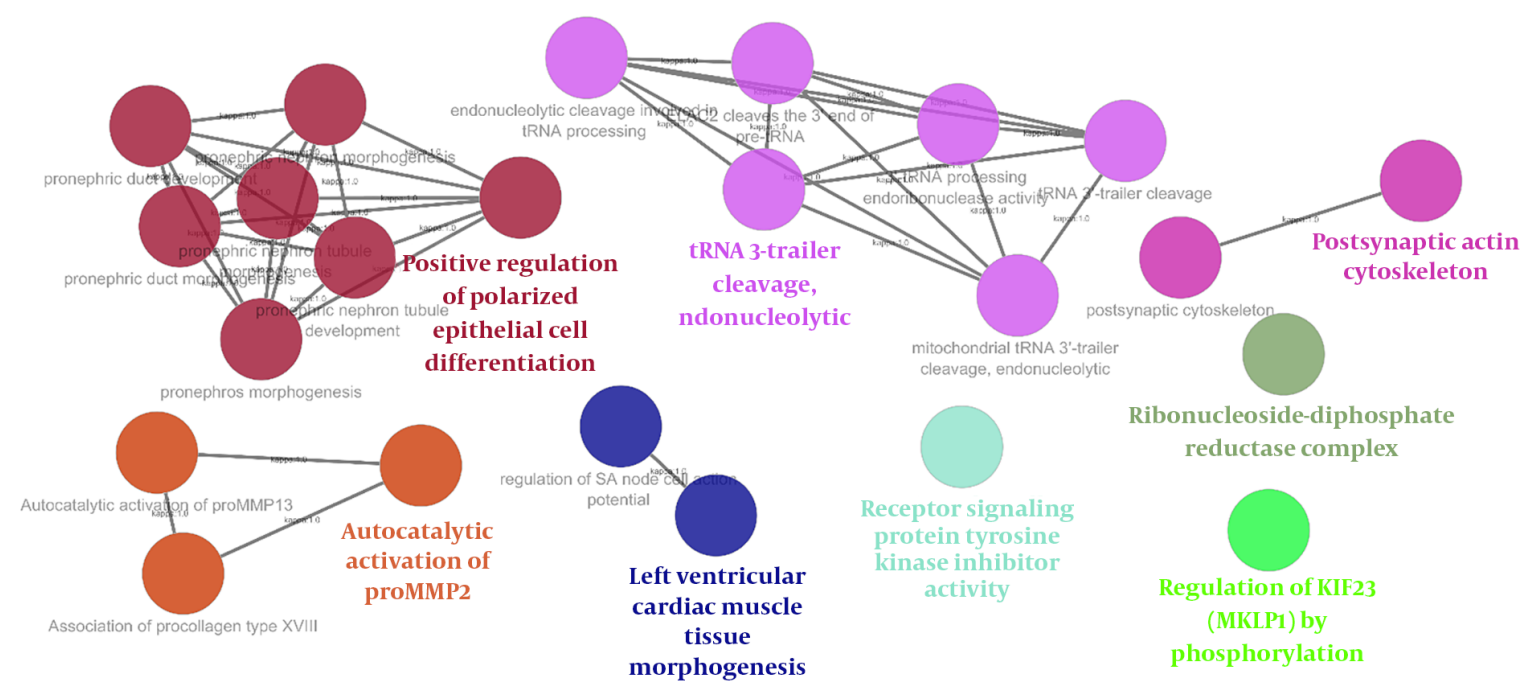

Figure 3. Eight significant $(\mathrm{P}<0.05)$ biological term including classified biological processes, molecular function, cellular component, and biochemical pathways related to the 20 critical genes of astrocytoma grade III versus grade II are shown.

data was not shown). To screen the DEGs, the related genes were found among various databases. As it is shown in the Figure 2, the constructed network by adding 4,444 genes is a scale-free network. Investigations indicate that central nodes in the scale-free networks are important reagents to onset and development of diseases (17). Fifty hubs were identified for grade II to grade III transition.

Considering two important futures including physicochemical properties and the expression level of the 45 significant DEGs and 50 hubs led to select 20 common hub nodes and significant DEGs (Table 1 ). So, the determined 20 critical genes that are involved in transition process of the both grades are as follow: Zinc finger protein 765 , zinc finger protein 540, Zeste white 10 (ZW10) ZW10 interacting kinetochore protein, collagen type XVIII alpha 1 chain, protamine 2 , kinesin family member 23 , minichromosome maintenance 10, anaphase promoting complex subunit 7, NADPH oxidase activator 1 , ryanodine receptor 2, myozenin 3, myosin binding protein $\mathrm{C}$, fast type, keratin 17, zinc phosphodiesterase ELAC protein 2, Abelson helper integration site 1 , latent transforming growth factor beta binding protein 1, kaptin (actin binding protein), BEN domain containing 3, cysteine, histidine rich 1 , and hyaluronoglucosaminidase 2 . For better understanding the biological role and also importance of the top 15 critical genes based on degree value is investigated in the literature sources and briefly is discussed.

Zinc finger proteins (ZNFs) are a family of various proteins, who are characterized by different cellular function.
ZNFs can bind to DNA and proteins by various domains (18). The role of ZWINT in mitotic process in cell proliferation is reported and discussed. Deregulation of this gene in cancer cells is investigated and confirmed (19). The prominent role of ZNFs in cancer progression is highlighted by several researches (20). Myozenin 3 and myosin binding protein $\mathrm{C}$ belong to skeletal muscle cells and also intracellular complexes, which are responsible for contractile process (21). Collagen Type XVIII is a component of extracellular matrix in liver that its level in fibrotic damage, cirrhosis, and cancer is altered (22). Investigation has shown that human collagen type I $\alpha 1$ gene acts as growth suppressor in the glioma cells (23). Therefore, down-regulation of collagen type 1 in grade III astrocytoma relative to grade II (Table 1) can be interpreted based on absence or decrease of a growth suppressor reagent in the grade III astrocytoma patients versus grade IIs. The role of PRM2 and PRM1 in sperm production is investigated and confirmed (24). Investigation has shown over-expression of KIF23 and KIF11 in malignant pleural mesothelioma (MPM) as well as human MPM cell line (25). The presence of KRT17 in oral squamous cell carcinoma is confirmed via immunohistochemistry and cDNA array methods (26). NADH oxidase is introduced as an essential member of oxygen-derived radicals, which is related to the vascular walls (27). MCM10, the other critical gene, is involved in DNA unwinding. This protein, together with several elements as capillary morphogenesis gene (CMG) complex, administrates its biological role (28). The role of ELAC2 gene together with TGF $\beta /$ Smad-induced 
transcriptional response in prostate cancer promotion is investigated (29). RyR2 plays a critical role to couple of excitation-contraction in cardiomyocytes (30). One of the critical genes is AHI1, which is related to retinitis pigmentosa disease (31). ANAPC7 is complicated in mitotic progression in eukaryotes (32).

As it was discussed, most of the critical genes are involved in the regulation of DNA duplication and cell proliferation processes. Muscular compartment also was the highlighted cellular component related to the hub-DEGs.

Based on the results of gene ontology analysis, which is illustrated in the Figure 3, 8 classes of biological terms related to the 20 critical genes are identified. The main class is positive regulation of polarized epithelial cell differentiation. This finding consists of the discussed documents as references 10 to 22 . Finally, it seems that as like as the other kinds of cancers, the regulation of cell proliferation and DNA duplication are the key points of grade II to grade III conversion of astrocytoma.

\subsection{Conclusions}

Introducing 20 critical genes as a biomarker panel was related to conversion of grade II to grade III of astrocytoma includes; first a challenge of numerous proposed biomarker candidate, which implies more screening to achieve a few numbers of biomarkers, and second the numerous proposed reagents can be useful to experience effective therapeutic and diagnostic procedures. The introduced crucial genes are potentially biomarkers of transitional phase from grade II to III.

\section{Acknowledgments}

None declared.

\section{Footnotes}

Authors' Contribution: None declared.

Conflict of Interests: There is no conflict of interest.

Financial Disclosure: None declared.

Funding/Support: This project is supported by Shahid Beheshti University of Medical Sciences.

\section{References}

1. Wang J, Bettegowda C. Genomic discoveries in adult astrocytoma. Curr Opin Genet Dev. 2015;30:17-24. doi: 10.1016/j.gde.2014.12.002. [PubMed: 25616158]. [PubMed Central: PMC4859210].

2. Jones DT, Mulholland SA, Pearson DM, Malley DS, Openshaw SW, Lambert SR, et al. Adult grade II diffuse astrocytomas are genetically distinct from and more aggressive than their paediatric counterparts. Acta Neuropathol. 2011;121(6):753-61. doi: 10.1007/s00401-011-0810-6. [PubMed: 21327941].
3. Narsia N, Ramagiri P, Ehrmann J, Kolar Z. Transcriptome analysis reveals distinct gene expression profiles in astrocytoma grades II-IV. Biomed Pap Med Fac Univ Palacky Olomouc Czech Repub. 2017;161(3):26171. doi: 10.5507/bp.2017.020. [PubMed: 28452381].

4. Sethi P, Treece J, Pai V, Onweni C, Rahman Z, Singh S. The mystery of multiple masses: A case of anaplastic astrocytoma. Cureus. 2017;9(6). e1384. doi: 10.7759/cureus.1384. [PubMed: 28775924]. [PubMed Central: PMC5522018].

5. Sarica FB, Cekinmez M, Tufan K, Sen O, Onal HC, Mertsoylu H, et al. Fiveyear follow-up results for patients diagnosed with anaplastic astrocytoma and effectiveness of concomitant therapy with temozolomide for recurrent anaplastic astrocytoma. Asian J Neurosurg. 2012;7(4):18190. doi: 10.4103/1793-5482.106650. [PubMed: 23559985]. [PubMed Central: PMC3613640].

6. Claus EB, Walsh KM, Wiencke JK, Molinaro AM, Wiemels JL, Schildkraut JM, et al. Survival and low-grade glioma: The emergence of genetic information. Neurosurg Focus. 2015;38(1). E6. doi: 10.3171/2014.10.FOCUS12367. [PubMed: 25552286]. [PubMed Central: PMC4361022].

7. Killela PJ, Pirozzi CJ, Reitman ZJ, Jones S, Rasheed BA, Lipp E, et al. The genetic landscape of anaplastic astrocytoma. Oncotarget. 2014;5(6):1452-7. doi: 10.18632/oncotarget.1505. [PubMed: 24140581]. [PubMed Central: PMC4039223].

8. MacDonald TJ, Pollack IF, Okada H, Bhattacharya S, Lyons-Weiler J. Progression-associated genes in astrocytoma identified by novel microarray gene expression data reanalysis. Methods $\mathrm{Mol}$ Biol. 2007;377:203-22. doi: 10.1007/978-1-59745-390-5_13. [PubMed: 17634619].

9. Kunkle BW, Yoo C, Roy D. Reverse engineering of modified genes by Bayesian network analysis defines molecular determinants critical to the development of glioblastoma. PLoS One. 2013;8(5). e64140. doi: 10.1371/journal.pone.0064140. [PubMed: 23737970]. [PubMed Central: PMC3667850].

10. Zamanian-Azodi M, Rezaei-Tavirani M, Rahmati-Rad S, Hasanzadeh $\mathrm{H}$, Rezaei Tavirani M, Seyyedi SS. Protein-protein interaction network could reveal the relationship between the breast and colon cancer. Gastroenterol Hepatol Bed Bench. 2015;8(3):215-24. [PubMed: 26328044]. [PubMed Central: PMC4553162].

11. Rezaei Tavirani M, Mansouri V, Rezaei Tavirani S, Hesami Tackallou S, Rostami - Nejad M. Gliosarcoma protein - protein interaction network analysis and gene ontology. Int J Cancer Manag. 2018;11(5). doi: 10.5812/ijcm.65701.

12. Zali H, Rezaei Tavirani M. Meningioma protein-protein interaction network. Arch Iran Med. 2014;17(4):262-72. [PubMed: 24724603].

13. Rezaei Tavirani M, Bashash D, Tajik Rostami F, Rezaei Tavirani S, Nikzamir A, Rezaei Tavirani M, et al. Celiac disease microarray analysis based on System Biology Approach. Gastroenterol Hepatol Bed Bench. 2018;11(3):216-24. [PubMed: 30013745]. [PubMed Central: PMC6040039].

14. Safari-Alighiarloo N, Rezaei-Tavirani M, Taghizadeh M, Tabatabaei SM, Namaki S. Network-based analysis of differentially expressed genes in cerebrospinal fluid (CSF) and blood reveals new candidate genes for multiple sclerosis. PeerJ. 2016;4. e2775. doi: 10.7717/peerj.2775. [PubMed: 28028462]. [PubMed Central: PMC5183126].

15. Rezaei-Tavirani M, Rezaei-Taviran S, Mansouri M, Rostami-Nejad M, Rezaei-Tavirani M. Protein-protein interaction network analysis for a biomarker panel related to human esophageal adenocarcinoma. Asian Pac J Cancer Prev. 2017;18(12):3357-63. doi: 10.22034/APJCP.2017.18.12.3357. [PubMed: 29286604]. [PubMed Central: PMC5980895].

16. Barabasi AL. Scale-free networks: A decade and beyond. Science. 2009;325(5939):412-3. doi: 10.1126/science.1173299. [PubMed: 19628854]. 
17. Safari-Alighiarloo N, Taghizadeh M, Rezaei-Tavirani M, Goliaei B, Peyvandi AA. Protein-protein interaction networks (PPI) and complex diseases. Gastroenterol Hepatol Bed Bench. 2014;7(1):17-31. [PubMed: 25436094]. [PubMed Central: PMC4017556].

18. Cassandri M, Smirnov A, Novelli F, Pitolli C, Agostini M, Malewicz $\mathrm{M}$, et al. Zinc-finger proteins in health and disease. Cell Death Discov. 2017;3:17071. doi: 10.1038/cddiscovery.2017.71. [PubMed: 29152378]. [PubMed Central: PMC5683310].

19. Ying H, Xu Z, Chen M, Zhou S, Liang X, Cai X. Overexpression of Zwint predicts poor prognosis and promotes the proliferation of hepatocellular carcinoma by regulating cell-cycle-related proteins. Onco Targets Ther. 2018;11:689-702. doi: 10.2147/OTT.S152138. [PubMed: 29440916]. [PubMed Central: PMC5800459].

20. Jen J, Wang YC. Zinc finger proteins in cancer progression. J Biomed Sci. 2016;23(1):53. doi: 10.1186/s12929-016-0269-9. [PubMed: 27411336]. [PubMed Central: PMC4944467].

21. Takada F, Vander Woude DL, Tong HQ, Thompson TG, Watkins SC Kunkel LM, et al. Myozenin: An alpha-actinin- and gamma-filaminbinding protein of skeletal muscle $\mathrm{Z}$ lines. Proc Natl Acad Sci $U$ S A. 2001;98(4):1595-600. doi: 10.1073/pnas.041609698. [PubMed: 11171996]. [PubMed Central: PMC29302].

22. Duncan MB, Yang C, Tanjore H, Boyle PM, Keskin D, Sugimoto H, et al. Type XVIII collagen is essential for survival during acute liver injury in mice. Dis Model Mech. 2013;6(4):942-51. doi: 10.1242/dmm.011577. [PubMed: 23580202]. [PubMed Central: PMC3701214].

23. Honma K, Miyata T, Ochiya T. Type I collagen gene suppresses tumor growth and invasion of malignant human glioma cells. Cancer Cell Int. 2007;7:12. doi: 10.1186/1475-2867-7-12. [PubMed: 17578585]. [PubMed Central: PMC1925056].

24. Cho C, Jung-Ha H, Willis WD, Goulding EH, Stein P, Xu Z, et al. Protamine 2 deficiency leads to sperm DNA damage and embryo death in mice. Biol Reprod. 2003;69(1):211-7. doi: 10.1095/biolreprod.102.015115. [PubMed: 12620939].

25. Kato T, Lee D, Wu L, Patel P, Young AJ, Wada H, et al. Kinesin fam- ily members KIF11 and KIF23 as potential therapeutic targets in malignant pleural mesothelioma. Int J Oncol. 2016;49(2):448-56. doi: 10.3892/ijo.2016.3566. [PubMed: 27279560].

26. Khanom R, Nguyen CT, Kayamori K, Zhao X, Morita K, Miki Y, et al. Keratin 17 is induced in oral cancer and facilitates tumor growth. PLoS One. 2016;11(8). e0161163. doi: 10.1371/journal.pone.0161163. [PubMed: 27512993]. [PubMed Central: PMC4981360].

27. Ambasta RK, Schreiber JG, Janiszewski M, Busse R, Brandes RP. Noxa1 is a central component of the smooth muscle NADPH oxidase in mice. Free Radic Biol Med. 2006;41(2):193-201. doi: 10.1016/j.freeradbiomed.2005.12.035. [PubMed: 16814099].

28. Looke M, Maloney MF, Bell SP. Mcm10 regulates DNA replication elongation by stimulating the CMG replicative helicase. Genes Dev 2017;31(3):291-305. doi: 10.1101/gad.291336.116. [PubMed: 28270517]. [PubMed Central: PMC5358725].

29. Noda D, Itoh S, Watanabe $Y$, Inamitsu M, Dennler S, Itoh F, et al ELAC2, a putative prostate cancer susceptibility gene product, potentiates TGF-beta/Smad-induced growth arrest of prostate cells. Oncogene. 2006;25(41):5591-600. doi: 10.1038/sj.onc.1209571. [PubMed: 16636667].

30. Blayney LM, Lai FA. Ryanodine receptor-mediated arrhythmias and sudden cardiac death. Pharmacol Ther. 2009;123(2):151-77. doi 10.1016/j.pharmthera.2009.03.006. [PubMed: 19345240]. [PubMed Central: PMC2704947]

31. Nguyen TT, Hull S, Roepman R, van den Born LI, Oud MM, de Vrieze $\mathrm{E}$, et al. Missense mutations in the WD40 domain of AHI1 cause nonsyndromic retinitis pigmentosa. J Med Genet. 2017;54(9):624-32. doi: 10.1136/jmedgenet-2016-104200. [PubMed: 28442542]. [PubMed Central: PMC5574394].

32. Ho AW, Garg AV, Monin L, Simpson-Abelson MR, Kinner L, Gaffen SL. The anaphase-promoting complex protein 5 (AnapC5) associates with A20 and inhibits IL-17-mediated signal transduction. PLoS One. 2013;8(7). e70168. doi: 10.1371/journal.pone.0070168. [PubMed: 23922952]. [PubMed Central: PMC3726431]. 